\title{
Real-time electronic patient evaluation of lymphedema symptoms, referral, and satisfaction: a cross-sectional study
}

\author{
Jennifer L. Nahum ${ }^{1}$, Mei R. Fu ${ }^{1,2}$, Joan Scagliola ${ }^{3}$, Martha Rodorigo ${ }^{1}$, Sandy Tobik ${ }^{1}$, Amber Guth ${ }^{4}$, \\ Deborah Axelrod ${ }^{4}$
}

${ }^{1}$ NYU Rory Meyers College of Nursing, New York, NY, USA; ${ }^{2}$ The Boston College William F. Connell School of Nursing, Chestnut Hill, MA, USA; ${ }^{3}$ Director of Nursing, NYU Clinical Cancer Center, New York, NY, USA; ${ }^{4}$ New York University School of Medicine, NYU Clinical Cancer Center, New York, NY, USA

Contributions: (I) Conception and design: MR Fu, J Scagliola, D Axelrod, A Guth, JL Nahum, M Rodorigo, S Tobik; (II) Administrative support: J Scagliola; (III) Provision of study materials or patients: D Axelrod, A Guth; (IV) Collection and assembly of data: JL Nahum, M Rodorigo, S Tobik; (V) Data analysis and interpretation: All authors; (VI) Manuscript writing: All authors; (VII) Final approval of manuscript: All authors.

Correspondence to: Mei R. Fu, PhD, RN, FAAN. Professor, Barry Family \& Goldman Sachs Endowed Professor, The Boston College William F. Connell School of Nursing, Chestnut Hill, MA, USA. Email: mei.r.fu@bc.edu.

Background: Lymphedema is a progressive and chronic illness. Early detection and treatment often lead to better clinical outcomes and improvement of patients' quality of life. Lymphedema symptoms can assist in detecting lymphedema. However, the use of patient-reported symptom evaluation is still limited in clinical practice. To address this gap in clinical practice, a metropolitan cancer center implemented an electronic patient evaluation of lymphedema symptoms (EPE-LE) to enable patients' real-time symptom report during patients' routine clinical visit while waiting to see their doctors in a waiting room. The purpose of this clinical project was to evaluate the usefulness of EPE-LE during patients' routine clinical visit.

Methods: A cross-sectional design was used. Participants were outpatient post-surgical breast cancer patients and clinicians who were involved in the EPE-LE implementation at a metropolitan cancer center of US. Data were collected during the three-month EPE-LE implementation, including patients' report of lymphedema symptoms, patient and clinician satisfaction, and referral to lymphedema specialists. Descriptive statistics were used for data analysis.

Results: During the three-month implementation, a total of 334 patients utilized the EPE-LE to report their lymphedema symptoms and 24 referrals to lymphedema specialists. Nearly all of the patients found that the EPE-LE was easy to use (91\%) and that they were satisfied with the EPE-LE for reporting lymphedema symptoms $(89 \%)$. The majority $(70 \%)$ of patients reported that the EPE-LE helped them to learn about symptoms related to lymphedema and encouraged them to monitor their symptoms. All clinicians (100\%) agreed that the use of the EPE-LE improved their lymphedema symptom assessment in post-surgical breast cancer patients; $75 \%$ reported that the EPE-LE increased their communication with patients related to lymphedema symptoms, $75 \%$ agreed they would recommend the EPE-LE for use at other cancer centers, and $75 \%$ reported that the information retrieved from the EPE-LE was helpful in evaluation of lymphedema.

Conclusions: The use of EPE-LE enhanced patients' real-time report of lymphedema symptoms, improved patient education on lymphedema symptoms, and helped clinicians for evaluation of lymphedema. The use of EPE-LE is an example how to implement evidence-based research into clinical practice that provides benefits for both patients and clinicians.

Keywords: Real-time; symptom; evaluation; lymphedema; electronic tablet; detection

Received: 23 March 2020; Accepted: 22 May 2020; Published: 20 April 2021.

doi: $10.21037 /$ mhealth-20-118

View this article at: http://dx.doi.org/10.21037/mhealth-20-118

(C) mHealth. All rights reserved. 


\section{Introduction}

Breast cancer-related lymphedema has been reported to affect $20-40 \%$ of women following breast cancer surgery (1-3). Lymphedema is defined as the swelling of a limb caused by the obstruction of the lymphatic system due to the treatment of breast cancer, such as surgery or radiation $(4,5)$. Lymphedema has profoundly negative effects on quality of life for breast cancer survivors $(6,7)$. Breast cancer survivors with lymphedema spend more days hospitalized, more days at outpatient appointments, and have more days absent from work annually compared to their nonlymphedema counterparts $(8,9)$. Follow-up appointments for lymphedema management, physical therapy, and equipment fitting (compression garments, bandages, creams) can create financial and economic burdens on breast cancer survivors with lymphedema (8). Early detection of lymphedema is necessary to preserve function of the affected limb and minimize symptoms $(10,11)$. Unfortunately, women treated for breast cancer commonly report being unaware that lymphedema as a possible consequence of breast cancer treatment (7). They report feeling as though health care professionals are not proactive in teaching lymphedema prevention and risk reduction strategies (7).

Patient-reported lymphedema symptoms are significantly associated with lymphedema and related inflammatory biophysiological mechanisms $(3,5)$. These symptoms include arm swelling, heaviness, tightness, firmness, pain, aching, soreness, tenderness, numbness, stiffness, tingling, burning, limb fatigue, limb weakness, seroma formation, breast swelling, chest wall swelling, limb hotness, blistering, as well as impaired limb mobility in the shoulder, arm, elbow, wrist, and fingers $(4,12,13)$. Research demonstrated that lymphedema symptoms can assist in detecting lymphedema defined by $>200 \mathrm{~mL}$ limb volume difference $(4,12)$. However, the use of patient-reported symptom evaluation is still limited in clinical practice to achieve early lymphedema detection as well as patient education on monitoring lymphedema symptoms.

To address this gap in clinical practice and promote early detection of lymphedema, a metropolitan cancer center in the US implemented an electronic patient evaluation of lymphedema symptoms (EPE-LE) to enable patients' real-time symptom report using a TouchScreen electronic tablet during patients' routine clinical visit while waiting to see their doctors in an Outpatient Breast Surgery Practice waiting room. The objective of this clinical project was to evaluate the usefulness of an EPE-LE to enable patients' real-time report of lymphedema symptoms using a TouchScreen electronic tablet during patients' routine clinical visit. The specific aims of this project were: (I) to assess the effectiveness of the use of EPE-LE to report lymphedema symptoms; (II) to evaluate patient and clinician satisfaction with the use of EPE-LE; and (III) to evaluate the referral rate to lymphedema therapy. We present the following article in accordance with the STROBE reporting checklist (available at http://dx.doi.org/10.21037/ mhealth-20-118).

\section{Methods}

\section{Study design}

A cross-sectional design was used for this clinical evaluation project to evaluate the usefulness of an EPE-LE to enable patients' real-time report of lymphedema symptoms using a TouchScreen electronic tablet during patients' routine clinical visit. The usefulness of EPE-LE was evaluated during the three-month implementation of EPE-LE in terms of patients' report of lymphedema symptoms, patient and clinician satisfaction with the use of the EPE-LE, and referral to lymphedema specialists.

\section{Setting}

This clinical project (i15-00696) was exempted by the institutional review board of a metropolitan university in New York City, US. The study was conducted in accordance with the Declaration of Helsinki (as revised in 2013). The setting was a National Cancer Institute (NCI) designated cancer center in New York City, US. The cancer center is part of a world-renowned academic medical center and the project occurred at the cancer center's Outpatient Breast Surgery Practice, which specializes in breast cancer treatment including co-morbidities associated with breast cancer treatment. The EPE-LE was implemented at the Outpatient Breast Surgery Practice from June $1^{\text {st }}$ to August $31^{\text {st }}, 2015$. During this time, patients were asked by the receptionist to complete EPE-LE and patient satisfaction survey when checking-in for their post-surgical breast cancer appointment with their doctors. Clinicians were invited to participate in the study to report their satisfaction of the EPE-LE from October $20^{\text {th }}$ to November $5^{\text {th }} 2015$.

Patients and clinicians recruited and informed that participation of patients in the EPE-LE to report their lymphedema symptoms and their satisfaction as well as 
clinicians' participation in evaluating the use of the EPELE was completely voluntary. If participants chose not to participate, there were no negative impacts on the standard patient care provided or clinician employment. Patients and clinicians were assured of confidentiality and only de-identified data were collected. Since this project only involved the use of a symptom assessment and satisfaction surveys as part of routine clinical visit for the patients and satisfaction surveys for clinicians, to ensure confidentiality, the completion of the symptoms assessment of the patients and satisfaction surveys of the patients and clinicians served as their consent to the study.

\section{Participants}

Participants were outpatient post-surgical breast cancer patients and clinicians who were involved in the EPELE implementation and allowed their patients to use the EPE-LE implementation at the Outpatient Breast Surgery Practice in a metropolitan cancer center of US. Inclusion criteria for patients were: (I) over age 18 years; (II) understanding English; (III) having had surgery for breast cancer (stage I-III). Patients were excluded if they were diagnosed with and treated for lymphedema and breast cancer metastasis (stage IV). Clinicians were eligible for the study if they were involved in the EPE-LE implementation and allowed their patients to use the EPELE implementation.

\section{Outcome variables and measures}

\section{Report of lymphedema symptoms using EPE-LE}

The Outpatient Breast Surgery Practice utilized researchevidenced, reliable, valid, patient-report symptom evaluation tool (12,14), The Breast Cancer and Lymphedema Symptom Index (BCLE-SEI), for patient to report lymphedema symptoms. EPE-LE hosted of 17 major lymphedema symptoms, including swelling in the affected body side (i.e., arm, hand, breast, and chest wall), heaviness, firmness, tightness, stiffness, pain/aching/soreness, numbness, tenderness, stiffness, burning, stabbing, tingling (pins and needles), fibrosis (skin toughness or thickness), seroma formation (i.e., pocket of fluid formed), limb fatigue, limb weakness, impaired mobility in the affected body side (i.e., shoulder, arm, elbow, and wrist/fingers). The EPE-LE used a response frame of "now" for all participants to ensure the real-time presence of symptoms.

Since $>9$ lymphedema symptoms differentiate patients with lymphedema defined by $>200 \mathrm{~mL}$ limb volume difference from patients without lymphedema, referral to lymphedema specialists using EPE-LE was based on the following criteria: (I) zero lymphedema symptoms-no risk of lymphedema, no action was needed; (II) one to nine lymphedema symptoms-minimal risk of lymphedema, no action was needed; (III) greater than nine lymphedema symptoms-high risk of lymphedema, referral to lymphedema specialists.

\section{Referral for lymphedema assessment and treatment}

The lymphedema referral number was obtained at the completion of the three-month implementation of EPELE from June 1, 2015 to August 31, 2015. We analyzed the number of lymphedema referrals and lymphedema referral rate by comparing the total number of referrals to lymphedema specialist to the number of patient visits to breast cancer surgeons involved in the EPE-LE implementation.

\section{Patient satisfaction}

We developed a six-question Likert-scale survey to evaluate patient satisfaction of using the EPE-LE. To limit recall bias, the satisfaction survey was presented at the end of each patient's EPE-LE symptom assessment. Patients had the option to answer or not answer the satisfaction questions.

\section{Clinician satisfaction}

Clinician satisfaction was evaluated using a five-question Likert-Scale online survey for the individual clinician satisfaction of the use of the EPE-LE. Clinicians were invited to participate in survey that was sent to their email and was available for completion at their convenience from October $20^{\text {th }}$ to November $5^{\text {th }} 2015$. On November 5, 2015 we obtained the de-identified results from the online survey.

\section{Procedures}

Prior to the implementation of the EPE-LE, we observed that ample time (about 30-60 minutes) was available between the patient's arrival to the clinic and the time when they met their healthcare providers, we determined that the wait time could be utilized for patients to report their lymphedema symptoms using a TouchScreen electronic tablet to enable patients' real-time report of symptoms during patients' clinical visit while waiting to see their doctors. When post-surgical breast cancer patients came to the clinic visit, during checked in, a clinical staff would 
offer the EPE-LE to patients. It took about 5-7 minutes for patients to complete the EPE-LE. Prior to the symptom assessment, patients were required to report if they had ever been diagnosed with or treated for lymphedema and breast cancer metastasis (stage IV). If so, they were excluded from participation in the EPE-LE. Once the patients finished the EPE-LE, the results were emailed to their providers. The providers had the opportunity to review the results prior to seeing the patient and was able to immediately address issues reported through the EPE-LE assessment.

\section{Data collection}

From June $1^{\text {st }}$ to August $31^{\text {st }} 2015$, a total of 372 patients at the Outpatient Breast Surgery Practice were offered the EPE-LE to report their lymphedema symptoms in realtime. Of 372 patients, 38 were already diagnosed with lymphedema and therefore excluded from the EPE-LE assessment. Data were obtained regarding the effectiveness of the EPE-LE to report lymphedema symptoms and generate lymphedema referrals upon three-month EPE-LE implementation from June 1 through August 31, 2015.

Data regarding patient satisfaction with the use of the EPELE were collected. To limit recall bias, the patient satisfaction survey was collected in real-time upon patients' use of EPELE, that is, patients used the EPE-LE to reported their realtime symptoms then they completed the survey questions regarding their satisfaction with the use of EPE-LE. The clinician satisfaction data were obtained de-identified using an online survey from October $20^{\text {th }}$ to November $5^{\text {th }} 2015$ and all four surgeons completed the survey.

\section{Data analysis}

All the patients' and clinicians' data were de-identified and obtained upon the three-month implementation of EPE-LE. Patients' report of lymphedema symptoms was analyzed using descriptive statistics. We calculated how many patients used the EPE-LE and how many patients reported: (I) zero lymphedema symptoms; (II) one to nine lymphedema symptoms; (III) greater than nine lymphedema symptoms. The lymphedema referral rate was calculated by comparing the total number of lymphedema referrals to the total number of patients evaluated by the breast surgeons during the three months after EPE-LE implementation. Patient satisfaction was analyzed using a six-question LikertScale survey via the EPE-LE. We calculated the number of questions answered by patients and their responses.
We considered an $80 \%$ completion rate and a score agree or strongly agree as acceptable for demonstrating patient satisfaction. Data regarding clinician satisfaction using a five-question Likert-Scale online survey were used to calculate the completion rate and the responses for each clinician. The evaluators considered an $80 \%$ completion rate and a score of agree or strongly agree as acceptable for demonstrating clinician satisfaction. There were no missing data from the patients regarding lymphedema symptom report but $6.7 \%$ missing from satisfaction surveys. There were no missing data from clinicians' satisfaction surveys.

\section{Results}

\section{Participants}

From June $1^{\text {st }}$ to August $31^{\text {st }} 2015$, a total of 372 patients at the Outpatient Breast Surgery Practice were offered the EPE-LE to report their lymphedema symptoms in realtime. Of the 372 patients offered the EPE-LE, 38 were already diagnosed with lymphedema and therefore excluded from the assessment. A total of 334 patients utilized the EPE-LE to report their lymphedema symptoms and of those patients 179 patients reported no symptoms, 155 patients reported 1-9 symptoms, and 20 patients reported greater than 9 symptoms. Four clinicians participated in the study, who were involved the EPE-LE implementation and allowed their patients to participant in the EPE-LE assessment (Figure 1).

\section{Patient referrals}

During the three-month EPE-LE implementation, 24 patients were seen at the outpatient lymphedema treatment clinic by lymphedema specialists. Those patents with referral to lymphedema specialists were those who reported 9 or more lymphedema symptoms. Before the EPE-LE implementation, there was no established methods of tracking patient referrals to lymphedema specialists by the Outpatient Breast Surgery Practice. The EPE-LE provided a method to automatically track which patients had been referred to a lymphedema specialist, thereby providing a vastly improved tracking system compared to the clinical practice prior to the EPE-LE implementation.

\section{Patient satisfaction}

Of the 334 patients who completed EPE-LE for real-time 

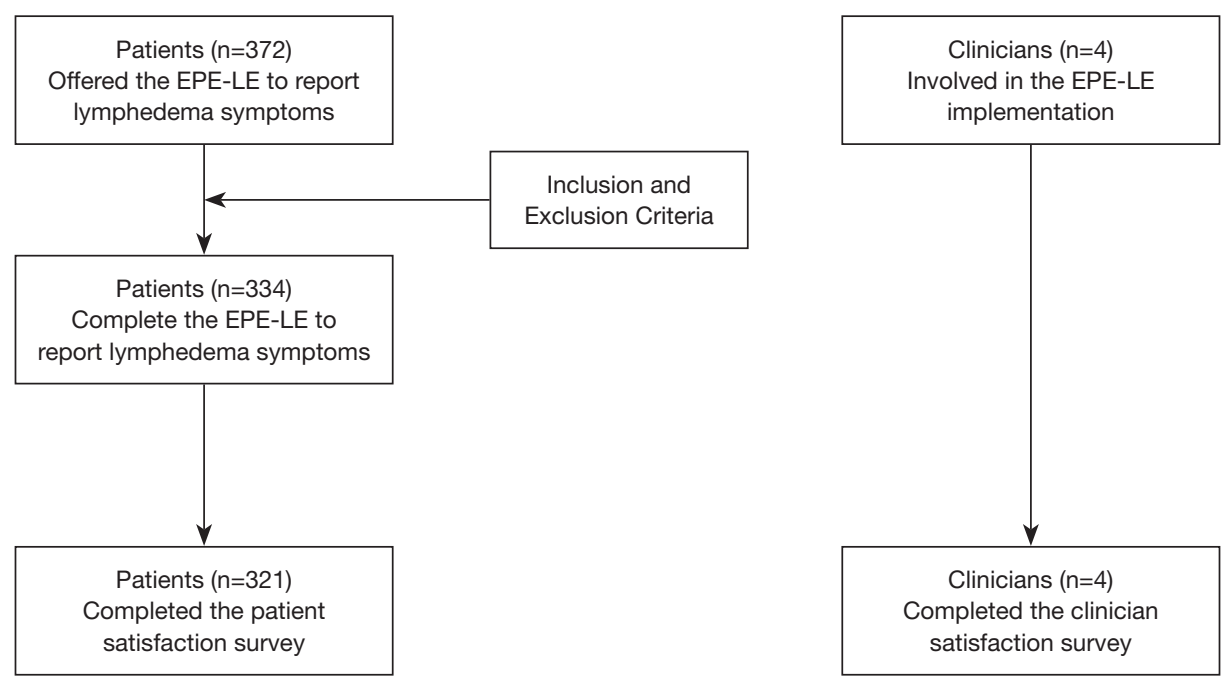

Figure 1 Flow diagram.

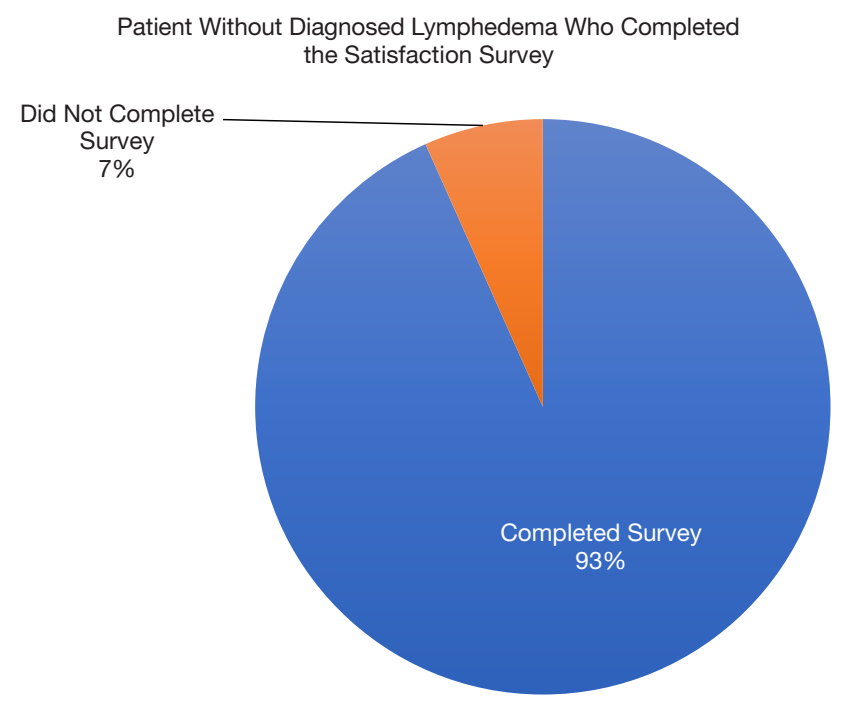

Figure 2 Completion of patient satisfaction survey.

lymphedema symptom evaluation, 321 (93.3\%) completed the patient satisfaction survey provided on the use of EPELE, surpassing the $80 \%$ completion rate goal (Figure 2). Over $70 \%$ of patients reported that utilizing the EPELE helped them to learn about symptoms related to lymphedema and that the EPE-LE encouraged them to monitor themselves for symptoms related to lymphedema. Nearly all of the patients found that the EPE-LE was easy to use $(91 \%)$ and that they were satisfied with the use of the EPE-LE for reporting lymphedema symptoms (89\%). While $13 \%$ of patients reported feeling indifferent about preferring the EPE-LE to the old-fashioned paper and pencil format, $84 \%$ of patients preferred this technology for symptom reporting. Eighty-seven percent of patient responders would recommend others use the EPE-LE to report their symptoms (Figure 3).

\section{Clinician satisfaction}

On November 5, 2015 we obtained results from the clinician questionnaires and evaluated the de-identified responses from all clinicians (Figure 4). We found that all four clinicians $(100 \%)$ agreed that the use of the TouchScreen EPE-LE improved their lymphedema symptom assessment in post-surgical breast cancer patients. In addition, $75 \%$ reported that utilizing the EPE-LE increased their communication with patients related to lymphedema symptoms, $75 \%$ were satisfied with the use of the EPE-LE in assessing lymphedema symptoms in postsurgical breast cancer patients, $75 \%$ agreed they would recommend the EPE-LE for use at other cancer centers, and $75 \%$ reported that the information retrieved from the EPE-LE was helpful in the evaluation of lymphedema in the post-surgical breast cancer patients.

\section{Discussion}

At the time of this project's analysis, there were no prior publications on evidence-based lymphedema self-assessment using a tablet evaluation in terms of its effect on patients' 


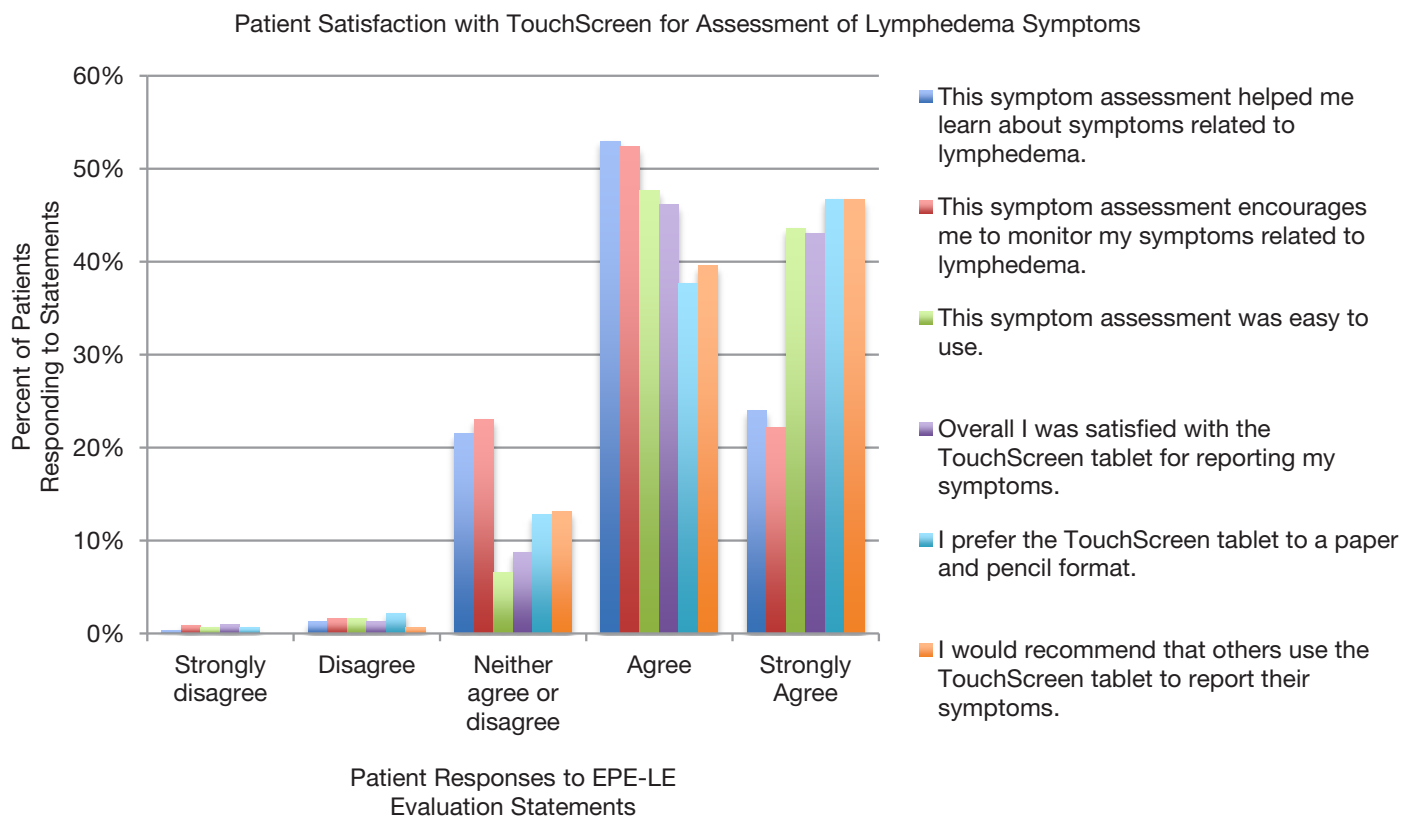

Figure 3 Patient Satisfaction with the TouchScreen EPE-LE. EPE-LE, electronic patient evaluation of lymphedema symptoms.

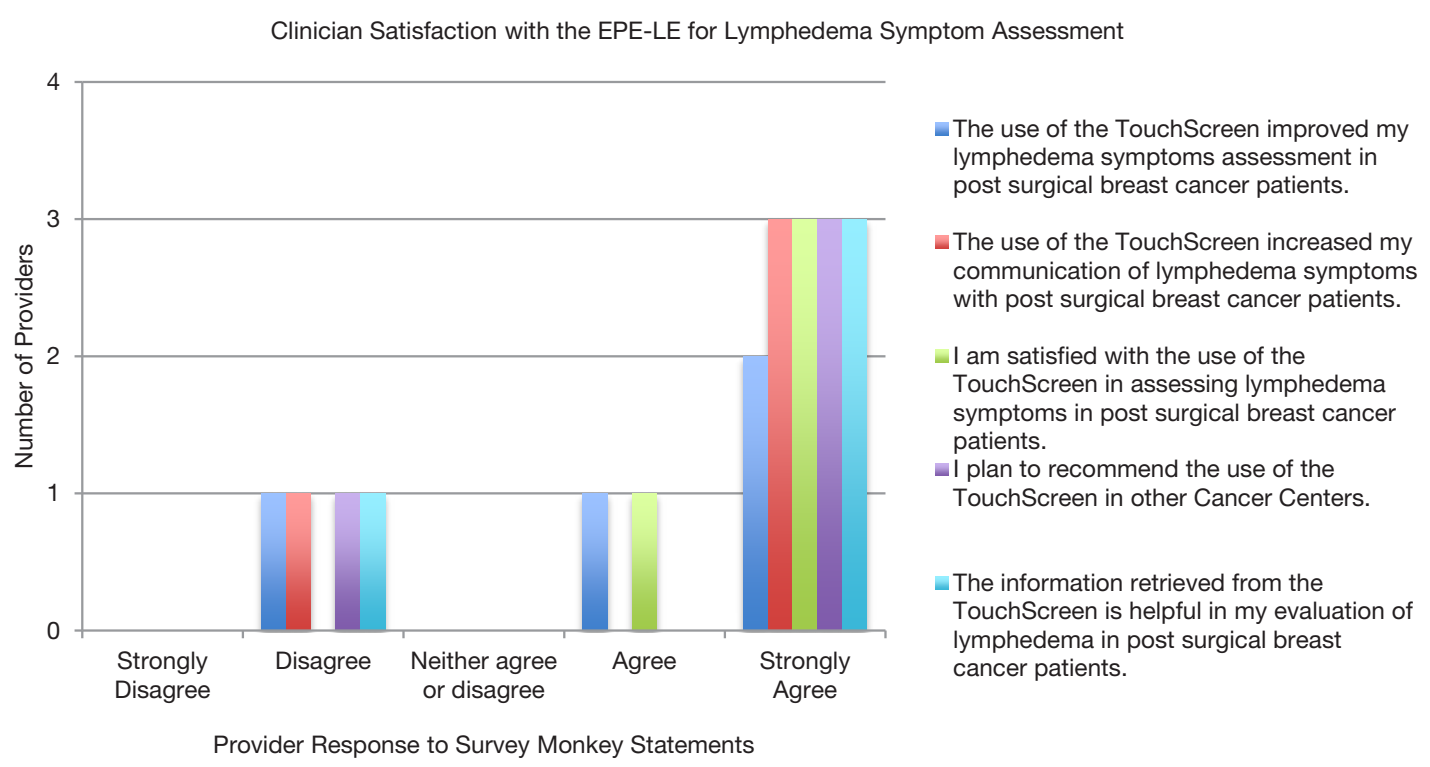

Figure 4 Clinician Satisfaction with the TouchScreen EPE-LE. EPE-LE, electronic patient evaluation of lymphedema symptoms.

lymphedema symptom reporting or lymphedema referral. We therefore consider this project to be the first in its kind and a benchmark for lymphedema symptom assessment utilizing a touchscreen tablet.

Research demonstrated that the effectiveness of using electronic tablet for patient-reported data (15), similarly the
EPE-LE is effective to enhance patients' real-time report of lymphedema symptoms and encourage patients to report and monitor their symptoms of lymphedema and help them to learn about lymphedema symptoms. Importantly, to sustain any changes in clinical practice, such as EPE-LE implementation, requires clinicians' buying and evaluation 
on whether the clinical change is effective to facilitate clinical practice. The clinicians who embraced the EPELE implementation reported being satisfied with the use of the EPE-LE to assess patients' lymphedema symptoms, improved clinicians' assessment of lymphedema symptoms in patients, and improved their communication with patients regarding lymphedema symptoms.

Lymphedema is a progressive and chronic illness and early detection and treatment often lead to better clinical outcomes and improvement of patients' quality of life $(2,11,16)$. Utilizing a cutoff point of more than 9 symptoms for referral to lymphedema specialists resulted in a referral rate of $7.1 \%$ ( 24 of 344 patients). It should be noted that utilizing only 9 or more lymphedema symptoms as cutoff may be an underestimate of lymphedema since nine symptoms is equivalent to moderate lymphedema defined by $>200 \mathrm{ml}$ limb volume differences $(4,12)$. As four or more symptoms can discriminate non-cancer healthy people from breast cancer survivors with lymphedema (12), it may be beneficial for patients who report four or more symptoms using the EPE-LE to be referred to lymphedema specialists, since those patients may have latent or mild lymphedema, if timely treatment occurs, latent and mild lymphedema can be reversed for those patients (2). If this more cautious step of using four or more symptoms as cut-off for this study, this would have led to an additional 34 referrals, a total of $78(23.5 \%)$ referrals. As $100 \%$ of patients with lymphedema report arm or hand swelling $(2-4,12)$, it may be beneficial for patients who report this symptoms using the EPE-LE to be referred to lymphedema specialists. Based on this cut-off, the EPE-LE assessment would have led to an additional 27 referrals, for a total of 81 referrals of 344 patients (24.3\%), compared to the 20 patients who were referred based on more than 9 symptom cut-off criterion. Notably, it was recognized that an organized and standardized lymphedema referral tracking system would be beneficial for clinical practice. Using the EPE-LE to record and track referrals is recommended. An automatic alert on the EPE-LE would inform clinicians when a patient scores higher than 4 on the symptom assessment and should prompt the provider to schedule a consult with a lymphedema specialist. Recent research also demonstrates that using machine learning procedures and well-trained classifier algorithms based on real-time symptom report can provide highly accurate detection of lymphedema (13). In future, effective machine learning algorithms for lymphedema detection may be incorporated into the EPE-LE to ensure more precision early detection of lymphedema based on real-time symptom report.

\section{Strengths}

The major strength of this project was its ability to use waiting time to assess patients for lymphedema, teach patients about lymphedema symptoms, and alert providers to the need for a lymphedema specialist referral for their post-surgical breast cancer patients. Another strength was the ease of use of the EPE-LE and satisfaction of patients and clinicians with the use of EPE-LE of reporting and monitoring for lymphedema symptoms. Another noted benefit of this work was the reduced stigma of post-surgical breast cancer lymphedema occurrence. It was noted that surgeons could be reluctant to mention lymphedema as a possible consequence of breast cancer surgery, possibly because of the thought that this may reflect poorly on their surgical skills. In addition, patients were reluctant to speak about side effects from their surgery likely due to the hope that the breast cancer had been removed and that there would be no further complications and the desire to believe that their surgeon was too skillful to allow any side effects to occur. In both cases the myth that poor surgery skills were the cause of lymphedema and the desire for no further health complications were barriers to the assessment, monitoring and education related to lymphedema. The EPE-LE leads to the de-stigmatization of these barriers and improved communication, assessment, and openness to teaching related to lymphedema.

\section{Limitations}

The EPE-LE in this project were administered in English language only. Having the EPE-LE available in additional languages may have increased patient completion and patient satisfaction scores. Patient demographics were not tracked and were not part of the project objectives. Lack of data to describe the patients' characteristics may affect the generalizability of the project. In the future, it would be particularly interesting to monitor patients' surgical history, age, ethnicity, and stage of breast cancer. It was identified that there is no organized referral tracking system at this site prior to the implementation of EPE-LE. We were unable to compare the referral rate after EPE-LE implementation to the referral rate before the implementation as this site did not have a standardized system to capture referrals. 
Utilizing the EPE-LE to track referrals would be beneficial for future monitoring of lymphedema detection. The financial cost of the EPE-LE as well as its implementation, including information technology (IT) and clerical staff involvement, is unknown to us. Despite this, the ability of EPE-LE to teach patients about lymphedema symptoms, enhance patients' self-monitoring of lymphedema symptoms, earlier detection, referral to prevent progression of symptoms and lymphedema may offset the cost. Future evaluation should include economic evaluation on both patient and institutional cost. Future studies should focus on implementation of the EPE-LE in a variety of clinical settings to increase the generalizability of using an EPELE to enable patients' real-time report of lymphedema symptoms during patients' routine clinical visit.

\section{Conclusions}

The use of EPE-LE is effective as nearly all of the patients found that the EPE-LE was easy to use (91\%) and that they were satisfied with using the EPE-LE for reporting lymphedema symptoms (89\%). The use of EPE-LE not only enhanced patients' real-time report of lymphedema symptoms that facilitated real-time referrals of patients at higher risk to lymphedema specialists but also led to clinicians and patients' satisfaction. Patients after breast cancer treatment face life-time risk of lymphedema $(2,4)$. The assessment of lymphedema risk and referral process based on patients' real-time report of lymphedema symptoms using the EPE-LE is effective for on-going monitoring of patients' symptom changes and evaluation of patients' lymphedema risk to ensure early detection of lymphedema. In addition, the use of EPE-LE also enhanced the patient education on lymphedema symptoms as over $70 \%$ of patients reported that utilizing the EPE-LE helped them to learn about symptoms related to lymphedema and that the EPE-LE encouraged them to monitor themselves for symptoms related to lymphedema. The use of EPE-LE is an example how to implement evidence-based research into clinical practice that provides benefits for both patients and clinicians.

\section{Acknowledgments}

The authors thank the nurses, doctors, and staff for their support for this project.

Funding: None.

\section{Footnote}

Provenance and Peer Review: This article was commissioned by the Guest Editor (Mei R Fu) for the series "Real-Time Detection and Management of Chronic Illnesses" published in mHealth. The article has undergone external peer review.

Reporting Checklist: The authors have completed the STROBE reporting checklist. Available at: http://dx.doi. org/10.21037/mhealth-20-118

Conflicts of Interest: All authors have completed the ICMJE uniform disclosure form (available at http://dx.doi. org/10.21037/mhealth-20-118). The series "Real-Time Detection and Management of Chronic Illnesses" was commissioned by the editorial office without any funding or sponsorship. MRF served as the unpaid Guest Editor of the series and serves as an unpaid editorial board member of mHealth. The authors have no other conflicts of interest to declare.

Ethical Statement: The authors are accountable for all aspects of the work in ensuring that questions related to the accuracy or integrity of any part of the work are appropriately investigated and resolved. The study was conducted in accordance with the Declaration of Helsinki (as revised in 2013). This clinical project (i15-00696) was exempted by the institutional review board of a metropolitan university in New York City, US.

Open Access Statement: This is an Open Access article distributed in accordance with the Creative Commons Attribution-NonCommercial-NoDerivs 4.0 International License (CC BY-NC-ND 4.0), which permits the noncommercial replication and distribution of the article with the strict proviso that no changes or edits are made and the original work is properly cited (including links to both the formal publication through the relevant DOI and the license). See: https://creativecommons.org/licenses/by-nc-nd/4.0/.

\section{References}

1. DiSipio T, Rye S, Newman B, et al. Incidence of unilateral arm lymphoedema after breast cancer: a systematic review and meta-analysis. Lancet Oncol 2013;14:500-15.

2. Cormier JN, Xing Y, Zaniletti I, et al. Minimal limb volume change has a significant impact on breast cancer survivors. Lymphology 2009;42:161-75. 
3. Fu MR, Conley YP, Axelrod D, et al. Precision assessment of heterogeneity of lymphedema phenotype, genotypes and risk prediction. Breast 2016;29:231-40.

4. Armer JM, Radina ME, Porock D, et al. Predicting breast cancer-related lymphedema using self-reported symptoms. Nurs Res 2003;52:370-9.

5. Stanton AW, Modi S, Mellor RH, et al. Recent advances in breast cancer-related lymphedema of the arm: lymphatic pump failure and predisposing factors. Lymphat Res Biol 2009;7:29-45.

6. O'Toole JA, Ferguson CM, Swaroop MN, et al. The impact of breast cancer-related lymphedema on the ability to perform upper extremity activities of daily living. Breast Cancer Res Treat 2015;150:381-8.

7. Fu MR, Rosedale M. Breast cancer survivors' experiences of lymphedema-related symptoms. J Pain Symptom Manage 2009;38:849-59.

8. Shih YC, Xu Y, Cormier JN, et al. Incidence, treatment costs, and complications of lymphedema after breast cancer among women of working age: a 2-year follow-up study. J Clin Oncol 2009;27:2007-14.

9. Stout NL, Pfalzer LA, Springer B, et al. Breast cancerrelated lymphedema: comparing direct costs of a prospective surveillance model and a traditional model of care. Phys Ther 2012;92:152-63.

doi: $10.21037 /$ mhealth-20-118

Cite this article as: Nahum JL, Fu MR, Scagliola J, Rodorigo M, Tobik S, Guth A, Axelrod D. Real-time electronic patient evaluation of lymphedema symptoms, referral, and satisfaction: a cross-sectional study. mHealth 2021;7:20.
10. Shah C, Arthur D, Riutta J, et al. Breast-cancer related lymphedema: a review of procedure-specific incidence rates, clinical assessment AIDS, treatment paradigms, and risk reduction. Breast J 2012;18:357-61.

11. Fu MR, Axelrod D, Guth AA, et al. Proactive approach to lymphedema risk reduction: a prospective study. Ann Surg Oncol 2014;21:3481-9.

12. Fu MR, Axelrod D, Cleland CM, et al. Symptom reporting in detecting breast cancer-related lymphedema. Breast Cancer (Dove Med Press) 2015;7:345-52.

13. Fu MR, Wang Y, Li C, et al. Machine learning for detection of lymphedema among breast cancer survivors. mHealth 2018;4:17.

14. Fu MR, Axelrod D, Guth AA, et al. mHealth self-care interventions: managing symptoms following breast cancer treatment. mHealth 2016;2:28.

15. Aiello EJ, Taplin S, Reid R, et al. In a randomized controlled trial, patients preferred electronic data collection of breast cancer risk-factor information in a mammography setting. J Clin Epidemiol 2006;59:77-81.

16. Kilbreath SL, Lee MJ, Refshauge KM, et al. Transient swelling versus lymphoedema in the first year following surgery for breast cancer. Support Care Cancer 2013;21:2207-15. 